\title{
Modelling and adaptive backstepping control for TX-1570 UAV path tracking
}

\author{
Julian Cayero*, Bernardo Morcego, Fatiha Nejjari \\ Department of Automatic Control (ESAII), Universitat Politècnica de Catalunya (UPC), Rambla de Sant Nebridi 22, 08222 Terrassa, Spain
}

\begin{abstract}
The methodology to design an aircraft longitudinal path-tracking controller is shown in this paper. First a thorough modelling process which involves the estimation of the aerodynamic and propulsion system structure and parameters is carried out. Next the Lyapunov based backstepping methodology is applied to design a non-linear controller for the aircraft longitudinal dynamics. The procedure used ensures convergence of the system to a reference and robustness in presence of modelling errors. The derived controller delivers manipulated inputs for a real plant and takes into account subsystem and model constraints. Finally, the performance of the controlled system tracking a realistic path is demonstrated in front of parameter uncertainty, unmodelled dynamics and adverse initial conditions.
\end{abstract}

Keywords: UAV, modelling, backstepping control, adaptive control

\section{Introduction}

Lately, unmanned air vehicles (UAVs) have been called to revolutionize the world of surveillance, security, fire-fighting and many other civil applications. This fact entails the necessity of flying over populated areas and in a non-segregated airspace. The laws that order the flight of UAVs in the civil airspace are not yet fully developed but organizations as the Joint Aviation Authorities, Eurocontrol and the European Aviation Safety Agency have set some guidelines for the future regulations, agreeing that the minimum requirements will be at least as restrictive as the present requirements for civil aircrafts in terms of safety, security and usage of the airspace [3]. Therefore, high levels of integrity and reliability are required to an UAV system in order to accomplish with the future established requirements. This leads to the necessity of control techniques that allow to determine the stability of the system under a consistent proven mathematical framework.

The backstepping technique $[7,8]$ applies a recursive procedure to the model of a dynamic system in order to stabilize it. The state space representation of the system needs to be compatible with the strict-feedback form. Through Lyapunov theory, an input profile is defined to take the system to a desired stable state. The principal benefit of backstepping is that it can be used in combination with adaptive techniques. Adaptive backstepping expands the domain of application to also deal with non-affine systems, in presence of modelling errors, external perturbations or even dramatic modifications of its dynamic equations (appropriate to model system external damage). Another useful improvement, named command filtered virtual control technique, can be added to incorporate in the

\footnotetext{
${ }^{*}$ Corresponding author

Email address: julen. cayero at upc.edu (Julian Cayero)
}

controller the knowledge about the actuator rate and magnitude limitations. It also simplifies the controller derivation process through an important effort saving related to the analytical mathematical development.

There is an increasing interest in the use of backstepping based techniques for the design of controllers for aircrafts and especially UAVs (see for example [4], [5], [13], [6] and [10], among others). In [4] backstepping was used to design an adaptive longitudinal flight controller that took into account the magnitude, rate and bandwidth constraints in the system's intermediate states and in the control surfaces. This work was extended later to control the airspeed and the flight-path angles of a tailless UAV [5]. In [12] a similar technique is used to develop a controller for velocity, angle of attack and roll around the stability axis of an F-16/MATV. In this case the aerodynamic nonlinear force and moment coefficients were parameterized by a neural network. In [13] a trajectory controller was derived for an F16 dynamic model. The aerodynamic forces and moments were approximated in this case by a B-spline neural network to cover the full flight envelope.

In some of the previous works it was supposed that the estimation of the input operator $g(x)$ was constant and full rank (recalling the affine non-linear general system description, $\dot{x}=$ $f(x)+g(x) u, g(x)$ is the equivalent to the input matrix $B$ in linear systems). That supposition is not realistic, taking into account the uncertain nature of the aerodynamic actions. In other cases where the adaptation process was used to cope with parameter uncertainty, the projection method was used to avoid the nullity of every column of $g(x)$, although linear independence between columns was not possible to guarantee.

However, the longitudinal problem addressed here reduces the estimation of $g(x)$ to a scalar. Therefore, the linearity dependence problem does not exist any more. In addition, the robustness of the controller in front of non-modelled terms and erroneous parameters is theoretically demonstrated and next the 
controller is tested by simulation with adverse initial conditions. It is shown that this control technique is suitable in cases when it is known beforehand that the model used to design the controller may be deficient.

The controllers proposed in the literature are often designed to produce output signals that are not direct inputs of the system, such as aerodynamic forces, moments or thrust forces in the case of aircrafts. When all the subsystem dynamics are not taken into account (i.e. the engine dynamics or servomotors) the resulting control laws may be sketchy and the simulation results may not show the real performance of the system.

In this paper, a realistic longitudinal model of the TX-1570 $\mathrm{UAV}$ is derived in the second section. This is accomplished with the Newton rigid-body equations of motion, an aerodynamic model obtained from simulation data and a semi-empirical model of the propulsion system. Next, the adaptive and robust backstepping procedure with command filtered virtual control modifications is used to derive a tracking controller. The controller is composed of velocity and path angle sub-controllers that supply real inputs of the system (voltage and elevator deflection signals), compatible with actuator operating ranges and model limits. Finally, simulation results show the tracking capabilities of the controller given a proposed path and the nice parameter adaptation controller skills. The controlled system can cope with initial modelling deviations introduced on purpose to test the robustness of this technique.

\section{Modelling of the fixed-wing aircraft TX-1570}

\subsection{System description}

The vehicle considered, called TX-1570 (Fig.1), is a fixedwing aircraft inspired from the commercial design of a highwing trainer of $1570 \mathrm{~mm}$ of span. The system is equipped with an EMax GT4020 brushless motor and with an APC-16 × 10E propeller. The aircraft is also equipped with a $400 \mathrm{mAh} 4$-cell lithium polymer battery that supplies up to $v_{\max }=14.8 \mathrm{~V}$ to the aircraft electronic circuit and propulsion system.

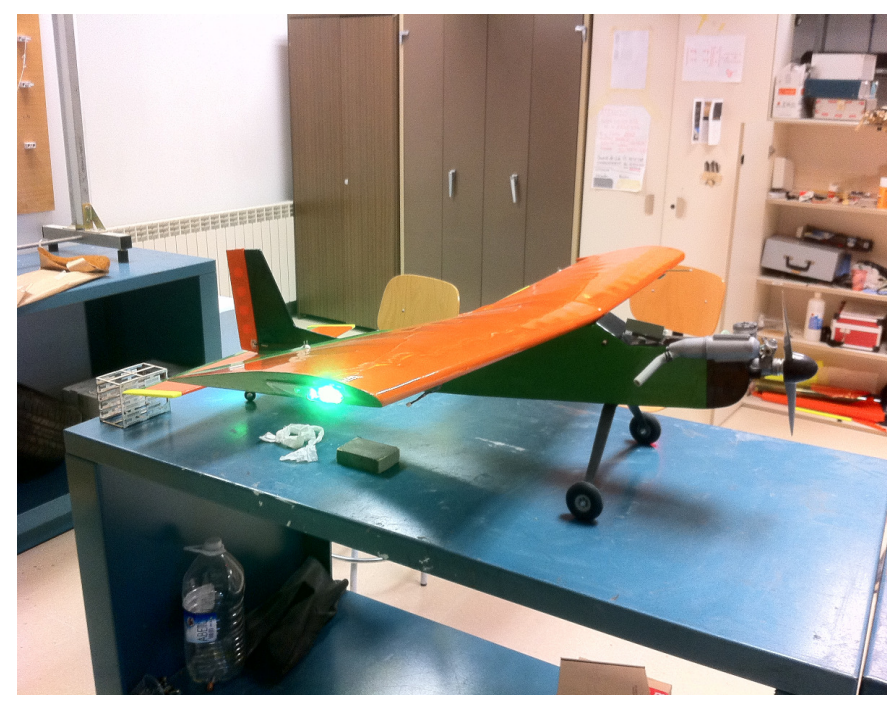

FIGURE 1: TX-1570 Aircraft.
The elevator presents a range of deflection $\delta_{e} \in[-30,30]$ deg. The servos that move the control surfaces are the High-Tech HS-7235MH, whose maximum deflection is larger than the one required by the elevator and their maximum turn-rate is $\omega_{s}=$ $10.48 \mathrm{rad} \mathrm{s}^{-1}$.

The main geometric characteristics of the TX-1570 used by the definition for the longitudinal model are given in Table. 1.

TABLE 1: TX-1570 Aircraft Characteristics.

\begin{tabular}{c|c|c|c}
\hline $\begin{array}{c}\text { Parame- } \\
\text { ter }\end{array}$ & Value & Units & Description \\
\hline$c$ & 0.287 & $\mathrm{~m}$ & Mean Aerodynamic Chord \\
$b$ & 1.570 & $\mathrm{~m}$ & Wing span \\
$S_{w}$ & 0.451 & $\mathrm{~m}^{2}$ & Wing area \\
$x_{c g}$ & 0.0957 & $\mathrm{~m}$ & Center of gravity longitudinal \\
& & & $\begin{array}{c}\text { position } \\
\text { Mass }\end{array}$ \\
$I_{y}$ & 2.89 & $\mathrm{~kg}$ & $\mathrm{k}$-Moment of inertia \\
\hline
\end{tabular}

\subsection{Equations of motion}

The equations of motion for a conventional fixed-wing aircraft can be derived through the rigid-body Newton equations in a non-inertial reference frame. When the motion is restricted to a longitudinal flight and the equations are applied over the body axes, the expressions become simpler because the inertia matrix becomes constant in time and the coupling between lateral and longitudinal inputs-outputs is removed. However, the resulting equations remain non-linear,

$$
\begin{gathered}
\dot{V}=\frac{1}{m}\left(F_{t} \cos (\alpha)-D-m g \sin (\gamma)\right), \\
\dot{\gamma}=\frac{1}{m V}\left(F_{t} \sin (\alpha)+L-m g \cos (\gamma)\right), \\
\dot{\alpha}=q-\frac{1}{m V}\left(F_{t} \sin (\alpha)+L-m g \cos (\gamma)\right), \\
\dot{q}=\frac{M}{I_{y}},
\end{gathered}
$$

being $V$ the velocity modulus, $\gamma$ the path angle, $\alpha$ the angle of attack and $q$ the pitch rate. $g$ represents the gravity vector, $F_{t}$ the thrust and $L, D$ and $M$ are the aerodynamic lift, drag and pitching torque, respectively. Fig. 2 illustrates the mentioned variables.

In order to complete the model, the external forces and torques have to be formulated as a function of the states $V, \gamma, \alpha$ and $q$ and the inputs $v$ and $\delta_{e}$.

\subsection{Aerodynamic model}

The drag $(D)$ and lift $(L)$ forces, formulated in wind axis, and the pitching moment $(M)$, formulated in body axis, have traditionally been expressed as a function of their non-dimensional aerodynamic coefficients, $c_{D}, c_{L}$ and $c_{M}$. This way it is possible 


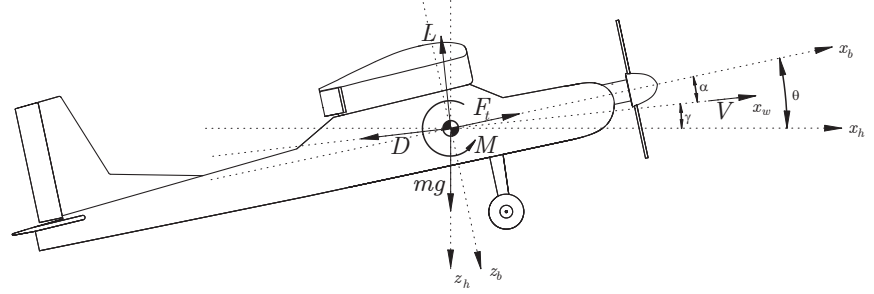

FIGURE 2: Definition of directions, angles, forces and torques for control. The subscript $w$ refers to the wind axis reference frame, the subscript $b$ refers to the body axis reference frame and the subscript $h$ refers to the local horizon axis reference frame.

to obtain the variation of aerodynamic actions with respect to the flight conditions, resulting in:

$$
\begin{aligned}
D & =\frac{1}{2} \rho V^{2} S c_{D}\left(\operatorname{Re}, \alpha, \dot{\alpha}, q, \dot{q}, \delta_{e}\right), \\
L & =\frac{1}{2} \rho V^{2} S c_{L}\left(\operatorname{Re}, \alpha, \dot{\alpha}, q, \dot{q}, \delta_{e}\right), \\
M & =\frac{1}{2} \rho V^{2} S c_{M}\left(\operatorname{Re}, \alpha, \dot{\alpha}, q, \dot{q}, \delta_{e}\right),
\end{aligned}
$$

where $\rho$ denotes the air density, $S$ represents the wing platform area and $R e$ is the Reynolds number. $c_{D}, c_{L}$ and $c_{M}$ are the aerodynamic coefficients for drag force, lift force and pitch moment, respectively.

In this paper the aerodynamic coefficients have been estimated with the help of the open-source tool XFLR5 [1]. This program allows the estimation of the aerodynamic coefficients considering several steady flight conditions using potential methods. Therefore, the dependency of the coefficients on $\dot{\alpha}$ and $q$ is not modelled. XFLR5 combines the potential theory with viscous estimations based on 2D profiles by means of XFOIL. The TX-1570 UAV aerodynamic model has been calculated by a 3D panel method, with a variation of $\alpha$ in the range of $[-10,10]$ deg and at a mean velocity of $19 \mathrm{~m} \mathrm{~s}^{-1}$, which is supposed to be representative of a mean $R e$ by several elevator deflection configurations in a range of $[-30,30] \mathrm{deg}$. The output results have been completed with an estimation of the parasite drag for the body [11, sec. 3.1.1], which is valid in the range $\left[1.8 \cdot 10^{6}, 3 \cdot 10^{6}\right]$ of the Reynolds number based on the length of the body.

The extracted data has been fitted through a linear regression process with a significance test for every parameter and for the whole model. The modified coefficient of determination, $\bar{R}$, has also been obtained as a quality estimator for the fitting. The results are shown in Eq. (5).

$$
\begin{gathered}
c_{L}=0.246+4.2113 \alpha+0.4755 \delta_{e} \\
c_{D}=0.048+0.1067 \alpha+0.3456 \alpha \delta_{e}+0.5067 \alpha^{2}+.1180 \delta_{e}^{2} \\
c_{M}=-0.0086-0.8203 \alpha+1.037 \delta_{e}
\end{gathered}
$$

Figs. 3-5 show the simulated points and the fitted surface as a function of the angle of attack and the elevator deflection.

\subsection{Propulsion Model of TX1570 UAV}

The aircraft propulsion system produces a thrust force vector that has a fixed direction with respect to the aircraft, and it is assumed to lie in the plane of symmetry of the aircraft.

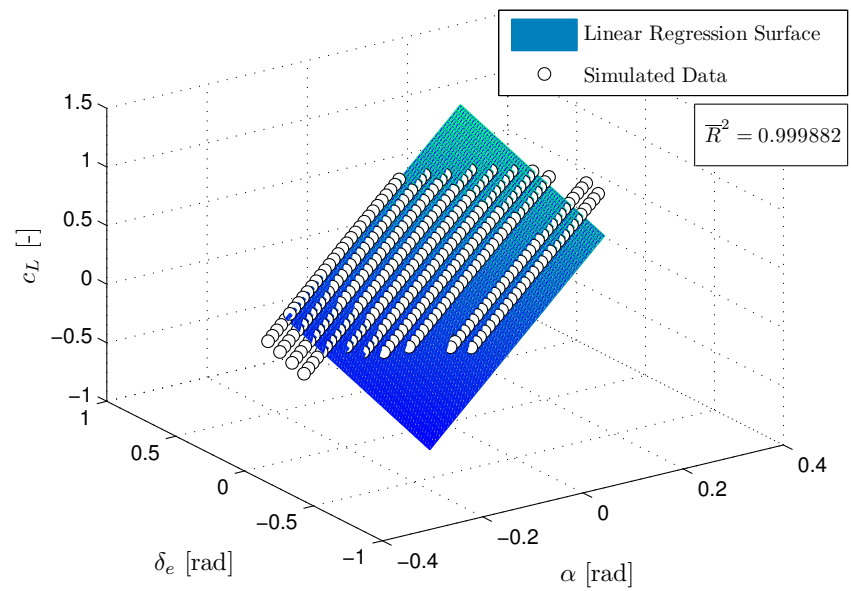

Figure 3: Adjustment $c_{L}$ vs. $\alpha$ and $\delta_{e}$.

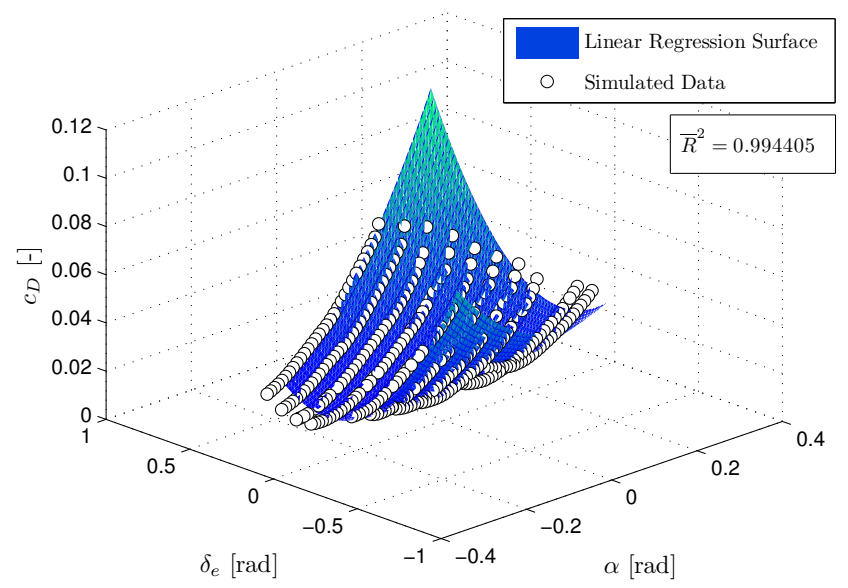

Figure 4: Adjustment $c_{D}$ vs. $\alpha$ and $\delta_{e}$.

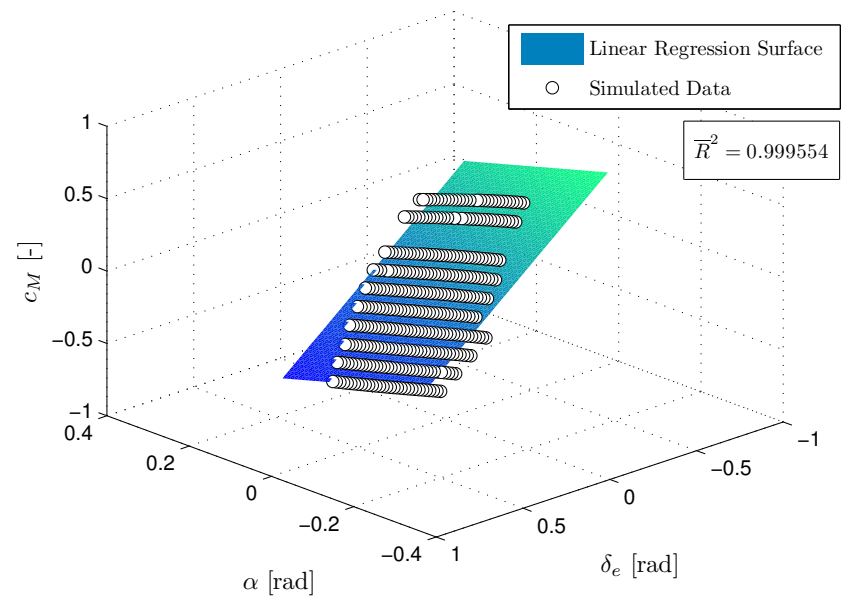

Figure 5: Adjustment $c_{M}$ vs. $\alpha$ and $\delta_{e}$.

The propulsion model of the TX1570 UAV consists of a combination of the engine model and the propeller model.

The TX-1570 has a single DC brushless electric motor with 
an internal resistance $R_{m}$ of mOhm, a no load current $i_{0}$ of $2 \mathrm{~A}$ and a constant $K_{V}$ of $620 \mathrm{rpmV}^{-1}$. The torque created by the motor and transmitted to the axis is given by:

$$
Q_{m}=\left(\left(v-\frac{\Omega}{K_{V}}\right) \frac{1}{R_{m}}-i_{0}\right) \frac{1}{K_{V}}
$$

where $v$ is the input voltage and $\Omega$ is the angular velocity.

The modelling of the aerodynamic forces that appear over the propeller has been approximated by recently published, [2], experimental data. In those experiments the values of thrust $\left(F_{t}\right)$ and power consumption $\left(P_{h}\right)$ are given as a function of the Reynolds number, the inflow velocity $(V)$ and the angular speed of the blade $(\Omega)$. The thrust and power are related to the nondimensional variables $c_{T}(\lambda, R e)$ and $c_{P}(\lambda, R e)$ by

$$
\begin{aligned}
& F_{t}(R e, \Omega, V)=\frac{1}{2} \rho(\Omega R)^{2} \pi R^{2} c_{T}(\lambda, R e), \\
& P_{h}(\operatorname{Re}, \Omega, V)=\frac{1}{2} \rho(\Omega R)^{3} \pi R^{2} c_{P}(\lambda, R e),
\end{aligned}
$$

where $\rho$ represents the density of the air, $R$ is the radius of the helix and $\lambda$ is the advance ratio $\lambda=\frac{V}{\Omega R}$.

Coefficients $c_{T}$ and $c_{P}$ were studied for the propeller APC$16 \times 10 \mathrm{E}$ chosen for the TX-1570 aircraft. They were considered in a wide range of Reynolds numbers that contemplates the possible operating speeds, proving to be sufficiently close to neglect the effect of the Reynolds number (see Fig. 6).

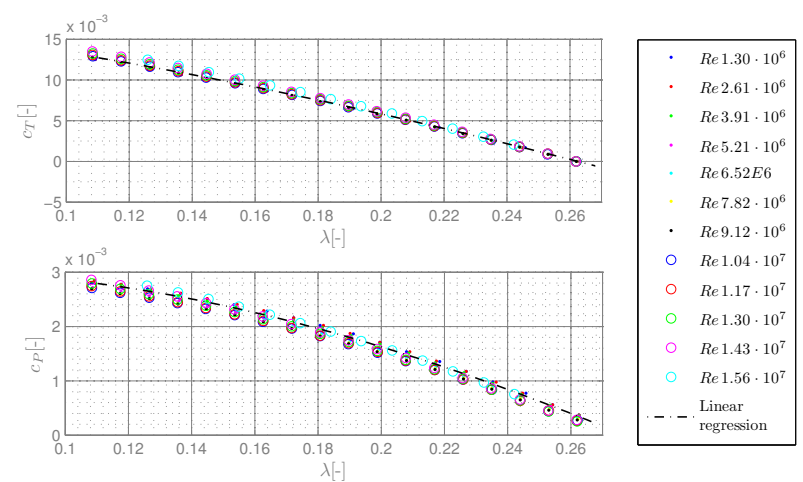

Figure 6: Experimental data $c_{T}$ y $c_{P}$ Propeller-16 $\times 10 \mathrm{E}$, with polynomial fitting.

The thrust and power coefficients have been successfully approximated by a third order polynomial equation under a linear regression process

$$
\begin{aligned}
& c_{T}=0.01828-0.03389 \lambda-0.16113 \lambda^{2}+0.09414 \lambda^{3}, \\
& c_{P}=0.00275-0.00830 \lambda-0.08221 \lambda^{2}+0.05676 \lambda^{3} .
\end{aligned}
$$

The individual models of the motor and the propeller are combined to predict the behaviour of the propulsion subsystem. The energy balance on the shaft establishes that the power delivered by the engine must be absorbed by the propeller. When the power balance is reached, the propeller and the shaft turn at the same speed of equilibrium $\Omega_{e q}$. Therefore, the equilibrium of the angular velocity depends on the flight velocity and the input voltage and can be found by solving the implicit equation: where the constants $c_{0}, c_{1}, c_{2}$ and $c_{3}$ are defined as

$$
\begin{array}{cc}
c_{0}=\frac{1}{2} \rho \pi R^{2} c_{P_{3}} V^{3} & c_{1}=\frac{1}{2} \rho \pi R^{2} c_{P_{2}} V^{2} R-\frac{v}{K_{V} R_{m}}+\frac{i_{0}}{K_{V}} \\
c_{2}=\frac{1}{2} \rho \pi R^{2} c_{P_{1}} V R^{2}+\frac{1}{K_{V}^{2} R_{m}} & c_{3}=\frac{1}{2} \rho \pi R^{2} c_{P_{0}} R^{3}
\end{array}
$$

After calculating the equilibrium angular velocity $\Omega_{e q}$ the parameter $\lambda_{e q}=\frac{V}{\Omega_{e q} R}$ is known and then the thrust can be recalculated according to Eq. (7). This process has been repeated for every combination of $v$ and $V$ after a discretization of $v \in$ $[0,15] \mathrm{V}$ and $V \in[12,30] \mathrm{m} \mathrm{s}^{-1}$ to derive the model. Finally a linear regression that represents the global model has been carried out to produce a continuous model.

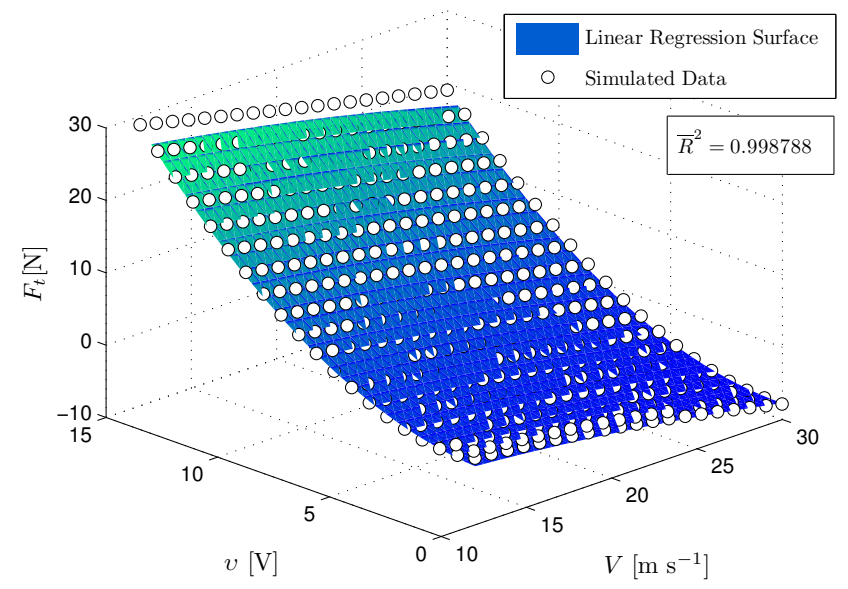

FIGURE 7: Fitting of the thrust surface.

$$
F_{t}(V, v)=-0.7881+0.3815 v-0.0128 V v-0.0077 V^{2}+0.12 v^{2}
$$

Again, the fitting proved to be significant for the model and for each one of its parameters. The estimator quality can be seen in Fig. 7 where the discrete data and the fitting surface are shown. The model has been calculated for a reference density $\rho_{0}=1,225 \mathrm{kgm}^{-3}$ therefore it is implicitly assumed that the variation of $F_{t}$ with the density is obtained through $F_{t}(\rho)=$ $F_{t} \frac{\rho}{\rho_{0}}$.

\section{Controller derivation}

This section describes the control law derivation for the longitudinal dynamics of the TX-1570 UAV in presence of error modelling and unmodelled linear and/or non-linear dynamics.

The control objective consists in solving the tracking problem for $V$ and $\gamma$ through the manipulation of the inputs $v$ and $\delta_{e}$ in the dynamic system represented by Eqs. (1-4). Two separate controllers are designed to solve this problem: the first one is in charge of following $V_{\text {ref }}$ (Eq. (1)) by producing a suitable voltage signal and the second one cares for $\gamma_{r e f}$ to be tracked (Eqs. (2-4)) by generating a $\delta_{e}$ command signal.

$$
c_{0}+c_{1} \Omega+c_{2} \Omega^{2}+c_{3} \Omega^{3}=0
$$




\subsection{Velocity module control}

The thrust model presented in section 2.4 is used to reproduce the real dynamics of the aircraft in the simulation results. As this model is not affine with respect to the input command and thus backstepping cannot be directly applied, it has been approximated by an an affine model to derive the controller.

$$
F_{t} \approx c_{T_{0}}+c_{T_{1}} V+c_{T_{2}} v+c_{T_{3}} V^{2}
$$

In addition, a simplification on the drag coefficient model has been done neglecting its dependence with respect to the elevator deflection

$$
c_{D} \approx c_{D_{0}}+c_{D_{1}} \alpha+c_{D_{2}} \alpha^{2}
$$

The approximation in the models introduce some inaccuracy that is compensated by the adaptive and robust terms present in the control law derived next. It must be noted that this is done at the expense of the controller and illustrates the fact that despite a perfect model is difficult to attain, the controller performs well.

With these assumed approximations, Eq. (1) can be rewritten as

$$
\dot{V}=f_{V}^{0}+\varphi_{f_{V}}^{T} \theta_{V}+\left(g_{V}^{0}+\varphi_{g_{V}}^{T} \theta_{V}\right) v+\phi_{V}^{T} \Delta_{V}
$$

where $\Delta_{V}$ is a vector of bounded unknown polynomial functions, $\phi_{V}$ is the set of parameters of those functions, selected as

$$
\phi_{V}^{T}=\left[\begin{array}{lllll}
V & V^{2} & \alpha & \alpha^{2} & v
\end{array}\right],
$$

and the rest of terms reproduce the results of the modelling process

$$
\begin{aligned}
& \varphi_{f_{V}}^{T}=\left[\begin{array}{lllllll}
a & a V & 0 & a V^{2} & b & b \alpha & b \alpha^{2}
\end{array}\right], \quad \varphi_{g_{V}}^{T}=\left[\begin{array}{lllllll}
0 & 0 & a & 0 & 0 & 0 & 0
\end{array}\right], \\
& \theta_{V_{0}}^{T}=\left[\begin{array}{lllllll}
c_{T_{0}}^{0} & c_{T_{1}}^{0} & c_{T_{2}}^{0} & c_{T_{3}}^{0} & c_{D_{0}}^{0} & c_{D_{1}}^{0} & c_{D_{2}}^{0}
\end{array}\right], \\
& \theta_{V}^{T}=\left[\begin{array}{lllllll}
\Delta c_{T_{0}}^{0} & \Delta c_{T_{1}}^{0} & \Delta c_{T_{2}}^{0} & \Delta c_{T_{3}}^{0} & \Delta c_{D_{0}}^{0} & \Delta c_{D_{1}}^{0} & \Delta c_{D_{2}}^{0}
\end{array}\right], \\
& f_{V}^{0}=\varphi_{f_{V}} \theta_{V_{0}}-g \sin (\gamma), \quad g_{V}^{0}=\varphi_{g_{V}} \theta_{V_{0}}, \\
& a=\frac{\cos (\alpha)}{m}, \quad b=-\frac{\rho V^{2} S}{2 m} \text {. }
\end{aligned}
$$

If an error $\tilde{\theta}$ in the estimation value of the parameters $\theta$ is considered, $\tilde{\theta}_{V}=\theta_{V}-\hat{\theta}_{V}$, the velocity dynamics can be expressed as

$$
\dot{V}=f_{V}^{0}+\varphi_{f_{V}}^{T} \hat{\theta}_{V}+\left(g_{V}^{0}+\varphi_{g_{V}}^{T} \hat{\theta}_{V}\right) v+\phi_{V}^{T} \Delta_{V}+\left(\varphi_{f_{V}}^{T}+\varphi_{g_{V}}^{T} v\right) \tilde{\theta}_{V}
$$

The voltage can be written as

$$
v=v^{0}+\left(v-v^{0}\right)
$$

in order to take into account the magnitude and rate limits imposed by the physical actuators. $v^{0}$ is the virtual control action, used from now on as the control action. The requested input voltage $v$ is obtained by filtering $v^{0}$ with a second order filter with internal limits to produce the desired restrictions. This filter is described in [5], and it is characterized by the natural frequency $\omega_{n}$ and the damping $\zeta$.

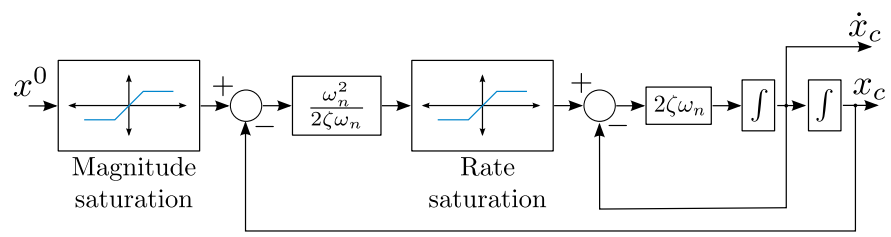

FIGURE 8: Second order filter scheme. The saturators are used to introduce the rate and magnitude limits.

Fig. 8 represents a generic filter that is used both here and in the attitude controller derivation. Signals $x^{0}$ and $x_{c}$ in the filter are assigned to signals $v^{0}$ and $v$ in the controller, respectively. $\dot{x}_{c}$ is not used.

Accordingly, the equation that describes the dynamics of velocity is given by

$$
\begin{aligned}
\dot{V}= & f_{V}^{0}+\varphi_{f_{V}}^{T} \hat{\theta}_{V}+\left(g_{V}^{0}+\varphi_{g_{V}}^{T} \hat{\theta}_{V}\right) v^{0}+\left(g_{V}^{0}+\varphi_{g_{V}}^{T} \hat{\theta}_{V}\right)\left(v-v^{0}\right) \\
& +\phi_{V}^{T} \Delta_{V}+\left(\varphi_{f_{V}}^{T}+\varphi_{g_{V}}^{T} v\right) \tilde{\theta}_{V} .
\end{aligned}
$$

Velocity tracking is the main objective, therefore it is convenient to define the velocity tracking error

$$
z_{V}=V-V_{r e f}
$$

and the compensated error

$$
\bar{z}_{V}=V-V_{r e f}-\xi_{V} .
$$

$\xi_{V}$ is the output of a stable filter that is necessary when the command filtered virtual control technique is used, as it can be seen in the following.

A control Lyapunov function (CLF) can be defined as

$$
W_{V}=\frac{1}{2} \bar{z}_{V}^{2}+\frac{1}{2} \tilde{\theta}_{V}^{T} \Gamma_{V}^{-1} \tilde{\theta}_{V}
$$

its time derivative is

$$
\dot{W}_{V}=\bar{z}_{V} \dot{\bar{z}}_{V}-\tilde{\theta}_{V}^{T} \Gamma_{V}^{-1} \dot{\hat{\theta}}_{V}
$$

if $\theta_{V}$ is assumed to be constant.

In view of the above, there are three free variables $v^{0}, \dot{\xi}_{V}$ and the adaptation law $\dot{\hat{\theta}}_{V}$ that can be chosen to make $\dot{W}_{V} \leq 0$.

When the virtual control action and the dynamics of $\xi_{V}$ are selected as

$$
v^{0}=\frac{1}{g_{V}^{0}+\varphi_{g_{V}}^{T} \hat{\theta}_{V}}\left(-f_{V}^{0}-\varphi_{f_{V}}^{T} \hat{\theta}_{V}+\dot{V}_{r e f}-k_{V} z_{V}-\phi_{V}^{T} \kappa_{V} \phi_{V} \bar{z}_{V}\right)
$$

and

$$
\dot{\xi}_{V}=-k_{V} \xi_{V}+g_{V}^{0}\left(v-v^{0}\right)
$$

and the adaptation law is defined as,

$$
\dot{\hat{\theta}}_{V}=\operatorname{Proj}\left(\Gamma_{V}\left(\varphi_{f_{V}} \bar{z}_{V}+\varphi_{g_{V}} \bar{z}_{V} v\right)\right),
$$

the derivative of the CLF becomes

$$
\begin{aligned}
\dot{W}_{V} & =\bar{z}_{V}\left(-k_{V} \bar{z}_{V}+\phi_{V}^{T} \Delta_{V}-\phi_{V}^{T} \kappa_{V} \phi_{V} \bar{z}_{V}\right) \\
& =-k_{V} \bar{z}_{V}^{2}+\bar{z}_{V} \phi_{V}^{T}\left(-\kappa_{V} \phi_{V} \bar{z}_{V}+\Delta_{V}\right) \\
& =-k_{V} \bar{z}_{V}^{2}-\left(\bar{z}_{V} \phi_{V}^{T}-\frac{\Delta_{V}^{T}}{2} \kappa_{V}^{-1}\right) \kappa_{V}\left(\bar{z}_{V} \phi_{V}-\kappa_{V}^{-1} \frac{\Delta_{V}}{2}\right)+\frac{\Delta_{V}^{T}}{2} \kappa_{V}^{-1} \frac{\Delta_{V}}{2},
\end{aligned}
$$


leading to

$$
\dot{W}_{V} \leq-k_{V} \bar{z}_{V}^{2}+\frac{\Delta_{V}^{T}}{2} \kappa_{V}^{-1} \frac{\Delta_{V}}{2} .
$$

Since $\dot{W}_{V}<0$ outside the $\Omega_{V}$ compact region, defined by

$$
\Omega_{V}=\left\{\bar{z}_{V} \mid 4 k_{V} \bar{z}_{V}^{2} \leq \Delta_{V}^{T} \kappa_{V}^{-1} \Delta_{V}\right\},
$$

and $W_{V}$ is radially unbounded, the compensated errors are globally uniformly ultimately bounded, with ultimate bound the boundary of $\Omega_{V}$. Therefore $\Omega_{V}$ represents an asymptotic stable set. It must be noted that this region can be done arbitrarily small by selecting sufficiently high values for $\kappa_{V}>0$ and $k_{V}>0$. As $\xi_{V}$ is the output of a stable filter, it tends to zero for a large enough value of $k_{V}$, then $z_{V}$ is bounded and therefore the tracking is accomplished within a certain tunable margin. This argumentation also holds to demonstrate the boundedness of the $\theta_{V}$.

The projection operator, chosen as in [9], is used to preserve the controllability, avoiding the situation where $g_{V}^{0}=-\varphi_{g_{V}}^{T} \hat{\theta}_{V}$ in which the virtual control action becomes infinity.

$$
\operatorname{Proj}(\tau)=\left\{\begin{array}{lr}
\left(I-\frac{\nabla f \nabla f^{T}}{\nabla f^{T} \nabla f} f\right) \tau, & \text { if } f \geq 0 \text { and } f^{T} \tau>0 \\
\tau, & \text { in any other case }
\end{array}\right.
$$

with

$$
f=f(\hat{\theta})=\frac{|\hat{\theta}|^{2}-\left(\theta_{\max }-\epsilon\right)^{2}}{2 \epsilon \theta_{\max }-\epsilon^{2}},
$$

being $\theta_{\max }$ the boundary value for the parameter vector at which the denominator of the virtual control action has the singularity, and $\epsilon$ is the distance to $\theta_{\max }$ to start applying the projection operator.

\subsection{Attitude control}

The aim of this controller is to generate the appropriate $\delta_{e}$ input command to track the prescribed flight-path reference $\gamma_{\text {ref }}$.

Eqs.(2)-(4), describe the dynamics of the flight-path angle in strict feedback form and they can be equivalently rewritten as

$$
\begin{gathered}
\dot{\gamma}=f_{\gamma}^{0}+\beta_{\gamma} \varphi_{f_{\gamma}}^{T} \theta+\left(g_{\gamma}^{0}+\beta_{\gamma} \varphi_{g_{\gamma}}^{T} \theta\right) \alpha+\phi_{\gamma}^{T} \Delta_{\gamma}, \\
\dot{\alpha}=f_{\alpha}^{0}+\beta_{\alpha} \varphi_{f_{\alpha}}^{T} \theta+q+\phi_{\alpha}^{T} \Delta_{\alpha}, \\
\dot{q}=f_{q}^{0}+\beta_{q} \varphi_{f_{q}}^{T} \theta+\left(g_{q}^{0}+\beta_{q} \varphi_{g_{q}}^{T} \theta\right) \delta_{e}+\phi_{q}^{T} \Delta_{q},
\end{gathered}
$$

where

$$
\begin{aligned}
& c_{L}=c_{L_{0}}+c_{L_{\alpha}} \alpha+c_{L_{\delta_{e}}} \delta_{e}, \quad c_{M}=c_{M_{0}}+c_{M_{\alpha}} \alpha+c_{M_{\delta_{e}}} \delta_{e}, \\
& \theta_{0}^{T}=\left[\begin{array}{llllll}
c_{L_{0}}^{0} & c_{L_{\alpha}}^{0} & c_{L_{\delta_{e}}}^{0} & c_{M_{0}}^{0} & c_{M_{\alpha}}^{0} & c_{M_{\delta_{e}}}^{0}
\end{array}\right], \\
& \theta^{T}=\left[\begin{array}{llllll}
\Delta c_{L_{0}} & \Delta c_{L_{\alpha}} & \Delta c_{L_{\delta_{e}}} & \Delta c_{M_{0}} & \Delta c_{M_{\alpha}} & \Delta c_{M_{\delta_{e}}}
\end{array}\right], \\
& \beta_{\gamma}=\frac{\rho V S}{2 m}, \\
& \beta_{\alpha}=-\frac{\rho V S}{2 m}, \\
& \beta_{q}=\frac{\rho V^{2} S c}{2 I_{y}}, \\
& \varphi_{f_{\alpha}}=\left[\begin{array}{llll}
1 & \alpha & \delta_{e} & 0^{1 \times 3}
\end{array}\right]^{T}, \quad \varphi_{f_{q}}=\left[\begin{array}{llll}
0^{1 \times 3} & 1 & \alpha & 0
\end{array}\right]^{T}, \\
& f_{\gamma}^{0}=\frac{F_{t} \sin (\alpha)}{m V}+\beta_{\gamma} \varphi_{f_{\gamma}}^{T} \theta_{0}-\frac{g \cos (\gamma)}{V}, \quad f_{\alpha}^{0}=-\frac{F_{t} \sin (\alpha)}{m V}+\beta_{\alpha} \varphi_{f_{\alpha}}^{T} \theta_{0}+\frac{g \cos (\gamma)}{V}, \\
& f_{q}^{0}=\beta_{q} \varphi_{f_{q}}^{T} \theta_{0} \\
& \varphi_{g_{\gamma}}=\left[\begin{array}{lll}
0 & 1 & 0^{1 \times 4}
\end{array}\right]^{T} \text {, } \\
& \varphi_{g_{q}}=\left[\begin{array}{ll}
0^{1 \times 5} & 1
\end{array}\right]^{T} \text {, } \\
& g_{\gamma}^{0}=\beta_{\gamma} \varphi_{g_{\gamma}}^{T} \theta_{0}, \\
& g_{q}^{0}=\beta_{q} \varphi_{g_{q}}^{T} \theta_{0}, \\
& \phi_{\gamma}=\phi_{\alpha}=\phi_{q}=\left[\begin{array}{lll}
1 & \alpha & \delta_{e}
\end{array}\right]^{T} .
\end{aligned}
$$

It can be seen that $\gamma$ would track a pre-established path, $\gamma_{r e f}$, if a specific $\alpha=\alpha_{c}$ was given. Eq. (17) can be used to generate $\alpha_{c}$ if the correct angular velocity $q=q_{c}$ is given, and finally the velocity $q_{c}$ can be achieved by means of Eq. (18) through the input $\delta_{e}$.

Introducing the error $\tilde{\theta}=\theta-\hat{\theta}$, and the virtual control actions $\alpha^{0}, q^{0}$ and $\delta_{e}^{0}$ through

$$
\begin{gathered}
\alpha=\alpha^{0}+\left(\alpha_{c}-\alpha^{0}\right)+z_{\alpha}, \\
q=q^{0}+\left(q_{c}-q^{0}\right)+z_{q}, \\
\delta_{e}=\delta_{e}^{0}+\left(\delta_{e}-\delta_{e}^{0}\right),
\end{gathered}
$$

where $z_{\gamma}=\gamma-\gamma_{c}$ is the path angle tracking error, $z_{\alpha}=\alpha-$ $\alpha_{c}$ is the angle of attack tracking error and $z_{q}=q-q_{c}$ is the pitch velocity tracking error. The dynamics of the compensated errors defined by

$$
\begin{gathered}
\bar{z}_{\gamma}=\gamma-\gamma_{r e f}-\xi_{\gamma}, \\
\bar{z}_{\alpha}=\alpha-\alpha_{c}-\xi_{\alpha}, \\
\bar{z}_{q}=q-q_{c}-\xi_{q},
\end{gathered}
$$

can be expressed as

$$
\begin{aligned}
\dot{\bar{z}}_{\gamma}= & f_{\gamma}^{0}+\beta_{\gamma} \varphi_{f_{\gamma}}^{T} \hat{\theta}+\left(g_{\gamma}^{0}+\beta_{\gamma} \varphi_{g_{\gamma}}^{T} \hat{\theta}\right) \alpha^{0}+\left(g_{\gamma}^{0}+\beta_{\gamma} \varphi_{g_{\gamma}}^{T} \hat{\theta}\right)\left(\alpha_{c}-\alpha^{0}\right) \\
& +\left(g_{\gamma}^{0}+\beta_{\gamma} \varphi_{g_{\gamma}}^{T}\right) z_{\alpha}+\phi_{\gamma}^{T} \Delta_{\gamma}-\dot{\gamma}_{r e f}-\dot{\xi}_{\gamma}+\beta_{\gamma} \varphi_{f_{\gamma}}^{T} \tilde{\theta}+\beta_{\gamma} \varphi_{g_{\gamma}}^{T} \tilde{\theta} \alpha \\
\dot{\bar{z}}_{\alpha}= & f_{\alpha}^{0}+\beta_{\alpha} \varphi_{\alpha}^{T} \hat{\theta}+q^{0}+\left(q_{c}-q^{0}\right)+z_{q}-\dot{\alpha}_{c}-\dot{\xi}_{\alpha}+\phi_{\alpha}^{T} \Delta_{\alpha} \\
& +\beta_{\alpha} \varphi_{\alpha}^{T} \tilde{\theta} \\
\dot{\bar{z}}_{q}= & f_{q}^{0}+\beta_{q} \varphi_{f_{q}}^{T} \hat{\theta}+\left(g_{q}^{0}+\beta_{q} \varphi_{g_{q}} \hat{\theta}\right) \delta_{e}^{0}+\left(g_{q}^{0}+\beta_{q} \varphi_{g_{q}} \hat{\theta}\right)\left(\delta_{e}-\delta_{e}^{0}\right) \\
& +\phi_{q}^{T} \Delta_{q}+\beta_{q} \varphi_{f_{q}}^{T} \tilde{\theta}+\beta_{q} \varphi_{g_{q}} \tilde{\theta} \delta_{e}
\end{aligned}
$$

\section{First backstepping step}

In order to accomplish a partial stabilization of the compensated error $\bar{z}_{\gamma}$, the first unbounded and positive semi-definite CLF is proposed

$$
W_{1}=\frac{1}{2} \bar{z}_{\gamma}^{2}
$$

and its derivative is

$$
\begin{aligned}
\dot{W}_{1}=\bar{z}_{\gamma} \dot{\bar{z}}_{\gamma}= & \bar{z}_{\gamma}\left(f_{\gamma}^{0}+\beta_{\gamma} \varphi_{f_{\gamma}}^{T} \hat{\theta}+\left(g_{\gamma}^{0}+\beta_{\gamma} \varphi_{g_{\gamma}}^{T} \hat{\theta}\right) \alpha^{0}\right. \\
& +\left(g_{\gamma}^{0}+\beta_{\gamma} \varphi_{g_{\gamma}}^{T} \hat{\theta}\right)\left(\alpha_{c}-\alpha^{0}\right)+\left(g_{\gamma}^{0}+\beta_{\gamma} \varphi_{g_{\gamma}}^{T} \hat{\theta}\right) z_{\alpha} \\
& \left.+\phi_{\gamma}^{T} \Delta_{\gamma}-\dot{\gamma}_{r e f}-\dot{\xi}_{\gamma}+\beta_{\gamma} \varphi_{f_{\gamma}}^{T} \tilde{\theta}+\beta_{\gamma} \varphi_{g_{\gamma}}^{T} \tilde{\theta} \alpha\right) .
\end{aligned}
$$


$\alpha_{c}$ and $\dot{\alpha}_{c}$ can be obtained using the 2 nd order filter in Fig. 8. If $\alpha^{0}$, is chosen as

$\alpha^{0}=\frac{1}{g_{\gamma}^{0}+\beta_{\gamma} \varphi_{g_{\gamma}}^{T} \hat{\theta}}\left(-f_{\gamma}^{0}-\beta_{\gamma} \varphi_{f_{\gamma}}^{T} \hat{\theta}+\dot{\gamma}_{r e f}-k_{\gamma} z_{\gamma}-\phi_{\gamma}^{T} \kappa_{\gamma} \phi_{\gamma} \bar{z}_{\gamma}\right)-\xi_{\alpha}$,

and in addition

$$
\dot{\xi}_{\gamma}=-k_{\gamma} \xi_{\gamma}+\left(g_{\gamma}^{0}+\beta_{\gamma} \varphi_{g_{\gamma}}^{T} \hat{\theta}\right)\left(\alpha_{c}-\alpha^{0}\right),
$$

the derivative of the proposed CLF becomes

$$
\begin{aligned}
\dot{W}_{1}= & \bar{z}_{\gamma}\left(-k_{\gamma} \bar{z}_{\gamma}+\left(g_{\gamma}^{0}+\beta_{\gamma} \varphi_{g_{\gamma}}^{T} \hat{\theta}\right) \bar{z}_{\alpha}+\phi_{\gamma}^{T} \Delta_{\gamma}-\phi_{\gamma}^{T} \kappa_{\gamma} \phi_{\gamma} \bar{z}_{\gamma}\right. \\
& \left.+\beta_{\gamma} \varphi_{f_{\gamma}}^{T} \tilde{\theta}+\beta_{\gamma} \varphi_{g_{\gamma}}^{T} \tilde{\theta} \alpha\right) .
\end{aligned}
$$

$\dot{W}_{1}$ is partially negative semi-definite, with the exception of the terms $\bar{z}_{\gamma}\left(g_{\gamma}^{0}+\beta_{\gamma} \varphi_{g_{\gamma}}^{T} \hat{\theta}\right) \bar{z}_{\alpha}$ and $\bar{z}_{\gamma} \beta_{\gamma} \tilde{\theta}^{T}\left(\varphi_{f_{\gamma}}+\varphi_{g_{\gamma}} \alpha\right)$.

\section{Second backstepping step}

The second step involves the stabilization of $z_{\gamma}$ and $z_{\alpha}$. It is done by augmenting the proposed CLF by a term that considers the $\bar{z}_{\alpha}$ states.

$$
W_{2}=W_{1}+\frac{1}{2} \bar{z}_{\alpha}^{2}
$$

Its time derivative is

$$
\begin{aligned}
\dot{W}_{2}= & \dot{W}_{1}+\bar{z}_{\alpha} \dot{\bar{z}}_{\alpha} \\
= & \dot{W}_{1}+\bar{z}_{\alpha}\left(f_{\alpha}^{0}+\beta_{\alpha} \varphi_{f_{\alpha}}^{T} \hat{\theta}+q^{0}+\left(q_{c}-q^{0}\right)\right. \\
& \left.+z_{q}-\dot{\alpha}_{c}-\dot{\xi}_{\alpha}+\phi_{\alpha}^{T} \Delta_{\alpha}+\beta_{\alpha} \varphi_{f_{\alpha}}^{T} \tilde{\theta}\right) .
\end{aligned}
$$

In order to partially stabilize the system, $q^{0}$ is chosen as

$q^{0}=-f_{\alpha}^{0}-\beta_{\alpha} \varphi_{f_{\alpha}}^{T} \hat{\theta}+\dot{\alpha}_{c}-k_{\alpha} z_{\alpha}-\phi_{\alpha}^{T} \kappa_{\alpha} \phi_{\alpha} \bar{z}_{\alpha}-\left(g_{\gamma}^{0}-\beta_{\gamma} \varphi_{g_{\gamma}}^{T} \hat{\theta}\right) \bar{z}_{\gamma}-\xi_{q}$

$q_{0}$ is also filtered, returning the magnitude and rate limited pair $q_{c}$ and $\dot{q}_{c}$. Defining

$$
\dot{\xi}_{\alpha}=-k_{\alpha} \xi_{\alpha}+\left(q_{c}-q^{0}\right)
$$

the resulting derivative of $W_{2}$ is

$$
\begin{aligned}
\dot{W}_{2}= & \bar{z}_{\gamma}\left(-k_{\gamma} \bar{z}_{\gamma}+\phi_{\gamma}^{T} \Delta_{\gamma}-\phi_{\gamma}^{T} \kappa_{\gamma} \phi_{\gamma} \bar{z}_{\gamma}+\beta_{\gamma} \varphi_{f_{\gamma}}^{T} \tilde{\theta}+\beta_{\gamma} \varphi_{g_{\gamma}}^{T} \tilde{\theta} \alpha\right) \\
& +\bar{z}_{\alpha}\left(-k_{\alpha} \bar{z}_{\alpha}+\phi_{\alpha}^{T} \Delta_{\alpha}-\phi_{\alpha}^{T} \kappa_{\alpha} \phi_{\alpha} \bar{z}_{\alpha}+\bar{z}_{q}+\beta_{\alpha} \varphi_{f_{\alpha}}^{T} \tilde{\theta}\right) .
\end{aligned}
$$

In this process some of the terms that make $\dot{W}_{1}$ non negative semi-definite have been canceled and new terms that make $\dot{W}_{2}$ non negative semi-definite have appeared. Those terms will be canceled in the next backstepping step.

\section{Third backstepping step}

Finally, the third step determines the elevator deflection, $\delta_{e}$, and the adaptation law of $\hat{\theta}$ to globally stabilize the system. In this case, the CLF can be augmented again to include the $\bar{z}_{q}$ state and the error in the parameter vector $\tilde{\theta}$

$$
W_{3}=W_{2}+\frac{1}{2} \bar{z}_{q}^{2}+\frac{1}{2} \tilde{\theta}^{T} \Gamma^{-1} \tilde{\theta},
$$

whose derivative is

$$
\begin{aligned}
\dot{W}_{3}= & \dot{W}_{2}+\bar{z}_{q} \dot{\bar{z}}_{q}-\tilde{\theta}^{T} \Gamma^{-1} \dot{\hat{\theta}} \\
= & \dot{W}_{2}+\bar{z}_{q}\left(f_{q}^{0}+\beta_{q} \varphi_{f_{q}}^{T} \hat{\theta}+\left(g_{q}^{0}+\beta_{q} \varphi_{g_{q}} \hat{\theta}\right) \delta_{e}^{0}\right. \\
& \left.+\left(g_{q}^{0}+\beta_{q} \varphi_{g_{q}} \hat{\theta}\right)\left(\delta_{e}-\delta_{e}^{0}\right)+\phi_{q}^{T} \Delta_{q}+\beta_{q} \varphi_{f_{q}}^{T} \tilde{\theta}+\beta_{q} \varphi_{g_{q}} \tilde{\theta} \delta_{e}\right) \\
& -\tilde{\theta}^{T} \Gamma^{-1} \hat{\hat{\theta}} .
\end{aligned}
$$

$\delta_{e}^{0}$ is chosen to partially stabilize the system,

$$
\delta_{e}^{0}=\frac{1}{g_{q}^{0}+\beta_{q} \varphi_{g_{q}}^{T} \hat{\theta}}\left(-f_{q}^{0}-\beta_{q} \varphi_{f_{q}}^{T} \hat{\theta}-\bar{z}_{\alpha}+\dot{q}_{c}-k_{q} z_{q}-\phi_{q}^{T} \kappa_{q} \phi_{q} \bar{z}_{q}\right),
$$

and then it is filtered by the second order filter to limit its magnitude and rate, producing the input $\delta_{e}$. Stable dynamics for $\xi_{q}$ are defined by

$$
\dot{\xi}_{q}=-k_{q} \xi_{q}+\left(g_{q}^{0}+\beta_{q} \varphi_{g_{q}} \hat{\theta}\right)\left(\delta_{e}-\delta_{e}^{0}\right),
$$

and are substituted in Eq. (25),

$$
\begin{aligned}
\dot{W}_{3}= & -k_{\gamma} \bar{z}_{\gamma}^{2}-k_{\alpha} \bar{z}_{\alpha}^{2}-k_{q} \bar{z}_{q}^{2}+\bar{z}_{\gamma}\left(\phi_{\gamma}^{T} \Delta_{\gamma}-\phi_{\gamma}^{T} \kappa_{\gamma} \phi_{\gamma}\right) \\
& +\bar{z}_{\alpha}\left(\phi_{\alpha}^{T} \Delta_{\alpha}-\phi_{\alpha}^{T} \kappa_{\alpha} \phi_{\alpha}\right)+\bar{z}_{q}\left(\phi_{q}^{T} \Delta_{q}-\phi_{q}^{T} \kappa_{q} \phi_{q}\right) \\
& +\tilde{\theta}^{T}\left(-\Gamma^{-1} \hat{\hat{\theta}}+\beta_{\gamma} \varphi_{f_{\gamma}} \bar{z}_{\gamma}+\beta_{\gamma} \varphi_{g_{\gamma}} \bar{z}_{\gamma} \alpha+\beta_{\alpha} \varphi_{f_{\alpha}} \bar{z}_{\alpha}\right. \\
& \left.+\beta_{q} \varphi_{f_{q}} \bar{z}_{q}+\beta_{q} \varphi_{g_{q}} \bar{z}_{q} \delta_{e}\right) .
\end{aligned}
$$

Then the adaptation law of $\hat{\theta}$ can be chosen as

$\dot{\hat{\theta}}=\operatorname{Proj}\left(\Gamma\left(\beta_{\gamma} \varphi_{f_{\gamma}} \bar{z}_{\gamma}+\beta_{\gamma} \varphi_{g_{\gamma}} \bar{z}_{\gamma} \alpha+\beta_{\alpha} \varphi_{f_{\alpha}} \bar{z}_{\alpha}+\beta_{q} \varphi_{f_{q}} \bar{z}_{q}+\beta_{q} \varphi_{g_{q}} \bar{z}_{q} \delta_{e}\right)\right)$,

where the projection operator averts $g_{q}^{0}=-\beta_{q} \varphi_{g_{q}}^{T} \hat{\theta}$ or $g_{\gamma}^{0}=$ $-\beta_{\gamma} \varphi_{\gamma_{g}}^{T} \hat{\theta}$, preventing to lose control over variables $\alpha^{0}$ and $\delta_{e}^{0}$.

The definition of $\dot{\hat{\theta}}$ finally makes $\dot{W}_{3}$ negative semi-definite outside the region

$$
\begin{aligned}
\Omega= & \left\{\left\{\bar{z}_{\gamma}, \bar{z}_{\alpha}, \bar{z}_{q}\right\} \mid 4\left(k_{\gamma} \bar{z}_{\gamma}^{2}+k_{\alpha} \bar{z}_{\alpha}^{2}, k_{q} \bar{z}_{q}^{2}\right) \leq \Delta_{\gamma}^{T} \kappa_{\gamma}^{-1} \Delta_{\gamma}+\right. \\
& \left.\Delta_{\alpha}^{T} \kappa_{\alpha}^{-1} \Delta_{\alpha}+\Delta_{q}^{T} \kappa_{q}^{-1} \Delta_{q}\right\},
\end{aligned}
$$

whose dimension can be done small enough by selecting high enough values for the controller gains $k_{\gamma, \alpha, q}>0$ and $\kappa_{\gamma, \alpha, q}>$ 0 . Since in addition $W_{3}$ is positive definite and radially unbounded and $\xi_{\gamma, \alpha, q}$ tends to zero by design, global uniform ultimate boundedness for the errors and boundedness for the parameter vector $\theta$ is guaranteed.

The final controller diagram, in which the derived control actions are implemented, is shown in Fig.9.

\section{Simulation results}

Results of applying the derived controller by simulation are presented below. The initial values of the simplified model chosen to derive the controller are:

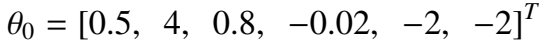

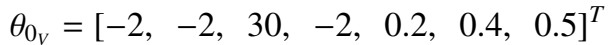




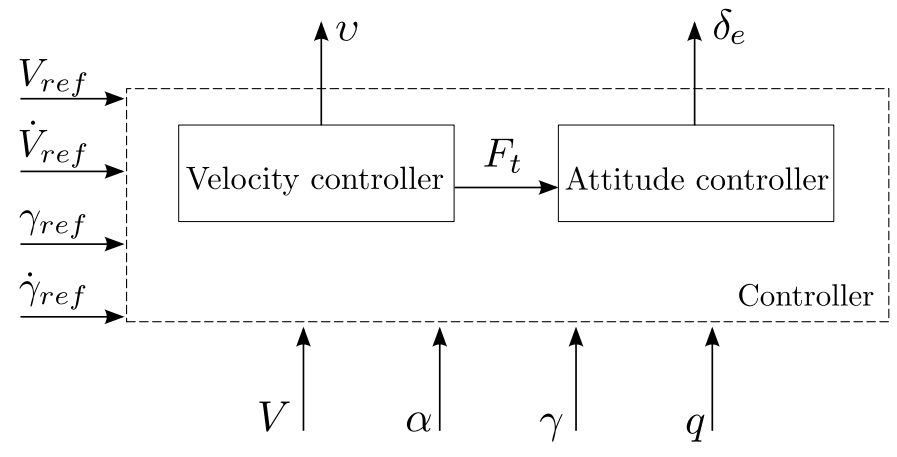

To make the simulation more realistic, the initial conditions applied to the aircraft are considered different from the references:

$$
\begin{gathered}
V_{0}=18 \mathrm{~m} / \mathrm{s} \quad \gamma_{0}=2 \mathrm{deg} \\
\alpha_{0}=-2 \mathrm{deg} \quad q_{0}=2.86 \mathrm{deg} / \mathrm{s}
\end{gathered}
$$

The references have been chosen attempting to show up the coupling between the response of tracking when the two controllers work simultaneously. These references are presented in Fig. 10. The derivative of the path angle reference has been set to 0 to avoid singularities, expecting that the adaptive control will be able to keep down the input discrepancies.
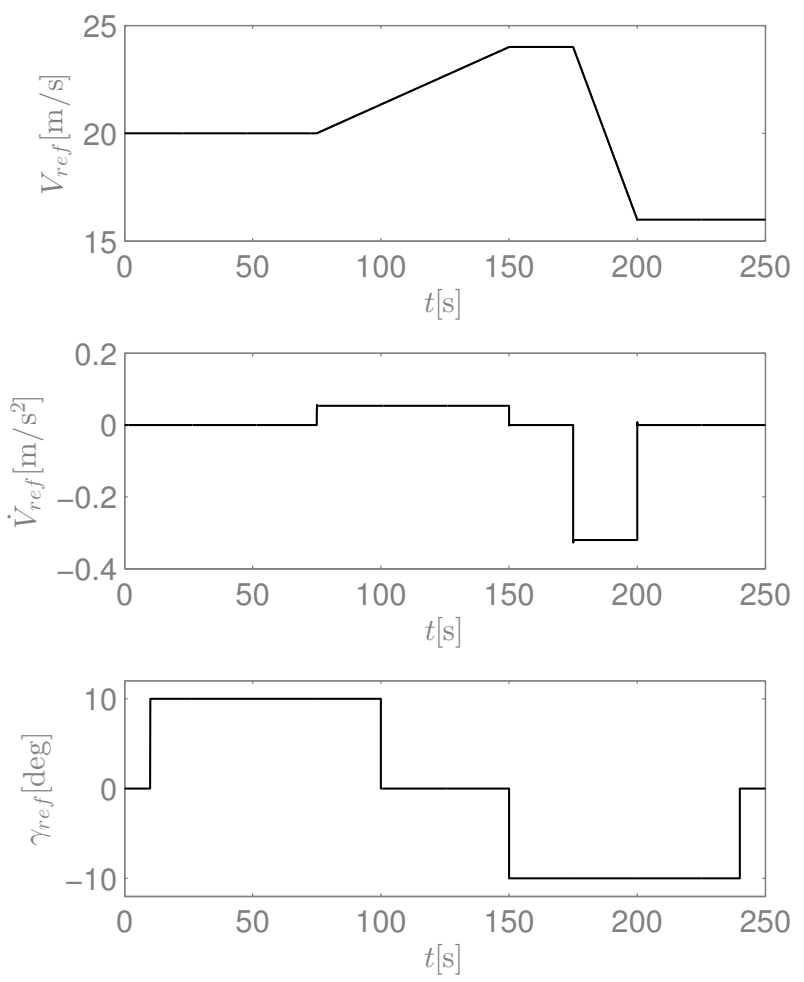

FIGURE 10: References as input for the controller.

The vectors containing the parameters of the incremental models, $\hat{\theta}$ and $\hat{\theta}_{V}$, have been initially set to zero.
The parameters chosen for tuning the controllers are given in Table 2.

\begin{tabular}{c|c}
\multicolumn{2}{c}{ TABLE 2: Controller Parameters } \\
\hline$k_{v}=3$ & $\kappa_{V}=20 I_{5}$ \\
$k_{\gamma}=2.5$ & $\kappa_{\gamma}=100 I_{3}$ \\
$k_{\alpha}=8$ & $\kappa_{\alpha}=200 I_{3}$ \\
$k_{q}=15$ & $\kappa_{q}=300 I_{3}$ \\
$\omega_{n_{v}}=300 \mathrm{rad} / \mathrm{s}$ & $\omega_{n_{\alpha}}=10.7 \mathrm{rad} / \mathrm{s}$ \\
$\omega_{n_{q}}=31.8 \mathrm{rad} / \mathrm{s}$ & $\omega_{n_{\delta}}=126.8 \mathrm{rad} / \mathrm{s}$ \\
$\zeta_{v}=0.7$ & $\zeta_{\alpha}=0.7$ \\
$\zeta_{q}=0.7$ & $\zeta_{\delta}=0.7$ \\
$\Gamma=200 I_{6}$ & $\Gamma_{V}=100 I_{7}$ \\
$\theta_{\max }=\min \left(\frac{g_{\gamma}^{0}}{\beta_{\gamma}}, \frac{g_{q}^{0}}{\beta_{q}}\right)$ & $\theta_{V_{\max }}=\frac{g_{V}^{0}}{a}$ \\
$\varepsilon=\frac{\theta_{\max }}{2}$ & $\varepsilon_{V}=\frac{\theta_{V_{\max }}}{2}$ \\
\hline
\end{tabular}

Figs. 11 and 12 show the tracking performance and the tracking errors. The maximum error in $V$ is $0.11 \mathrm{~m} / \mathrm{s}$ at $t=240.47 \mathrm{~s}$, where the setpoint of the flight-path angle returns to 0 after being at $-10 \mathrm{deg}$. This error represents less than the $1.5 \%$ of the the maximum excursion of $V$. The largest errors in $V$ appear when $\gamma_{\text {ref }}$ changes abruptly.

The maximum settling time of $\gamma$ (at $2 \%$ of the output) is $1.86 \mathrm{~s}$ at time $t=150 \mathrm{~s}$ when both $\gamma_{\text {ref }}$ and $V_{\text {ref }}$ change.
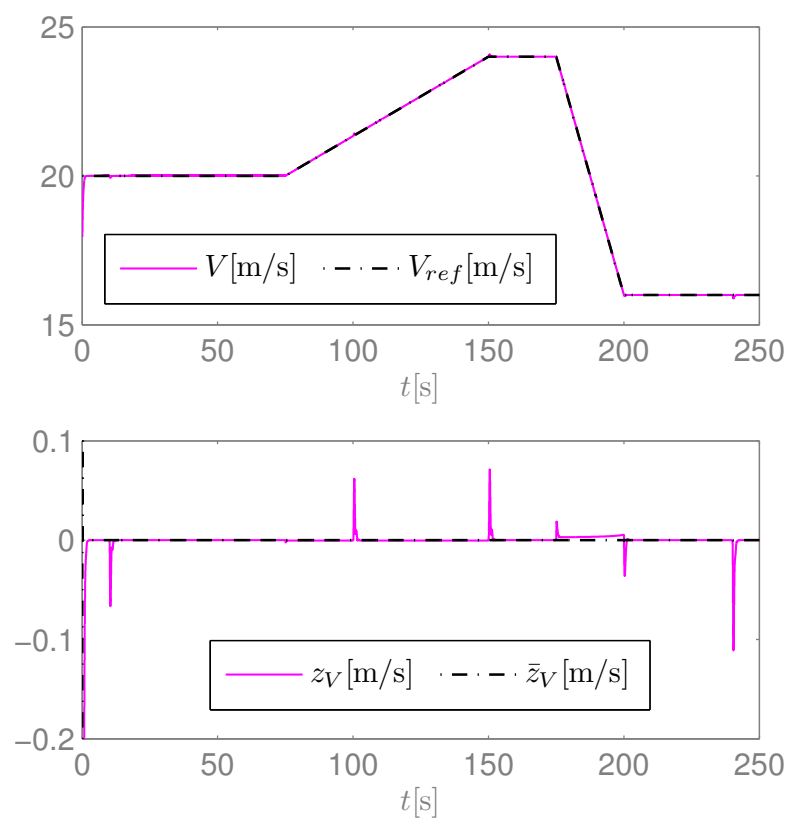

FIGURE 11: Tracking and error tracking of velocity reference.

In Fig. 13 the controller outputs are shown. It is important to note that the control actions satisfy the servo velocity restrictions, the maximum elevator's deflection restriction and the battery's voltage supply restriction. 

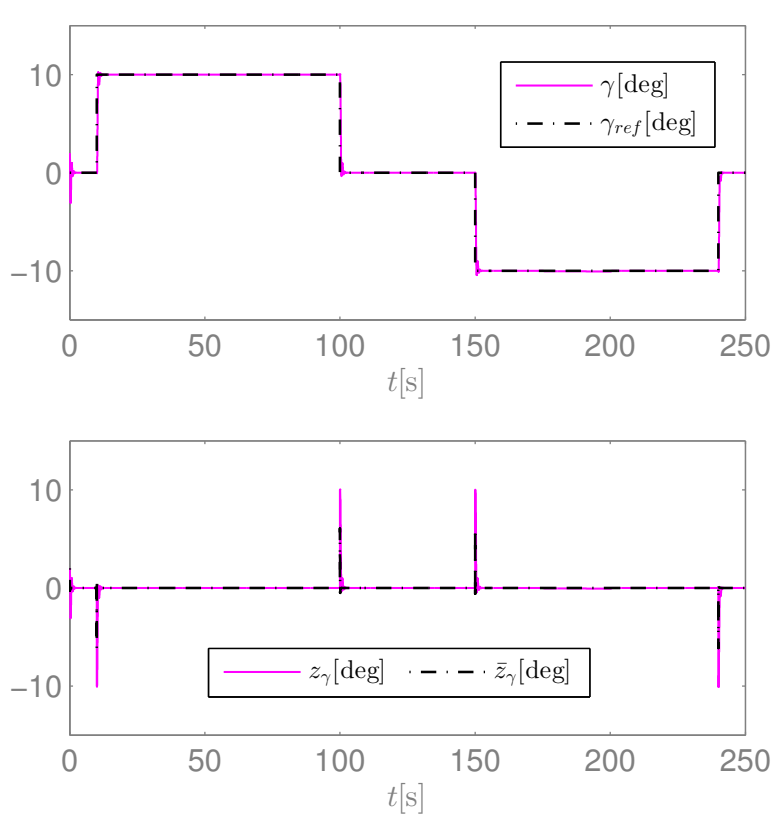

FIGURE 12: Tracking and error tracking of path angle reference.
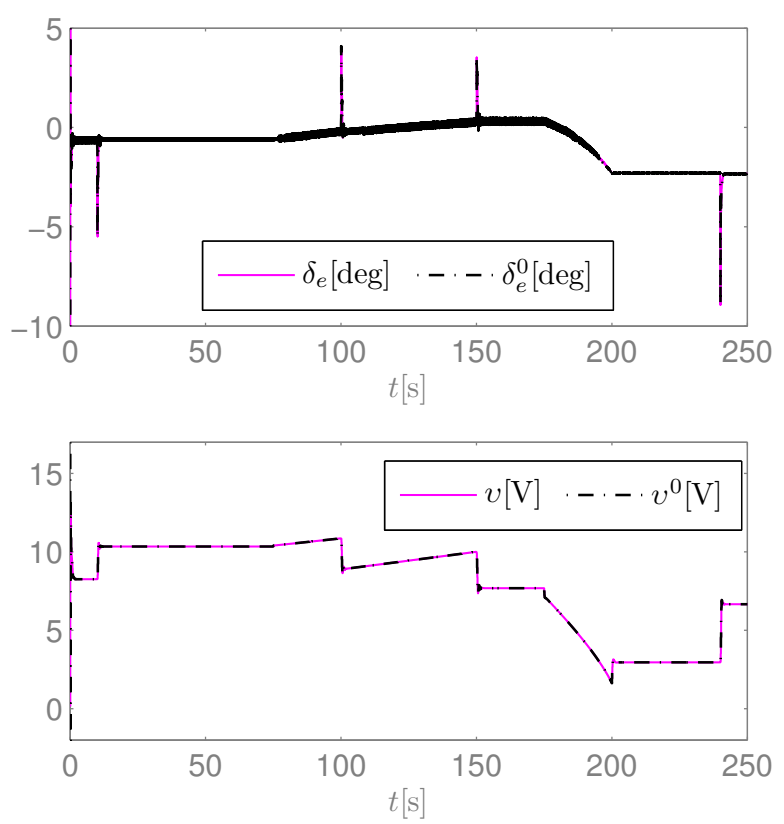

FIGURE 13: Output of the controller.

Figs. 14 and 15 show the evolution of $\alpha$ and $q$. It can be observed that $\alpha$ agrees with the limits of the model.

Finally, Figs. 16 and 17 show the evolution of the incremental models of the parameters of $c_{L}, c_{M}, F_{t}$ and $c_{D}$. It is noticeable that those parameters reach a stationary value. This is apparent for $c_{L}$ and $c_{M}$ but in the case of $F_{t}$ and $c_{D}$ it is the nonlinear damping terms that makes them reach a constant value after a transient period.
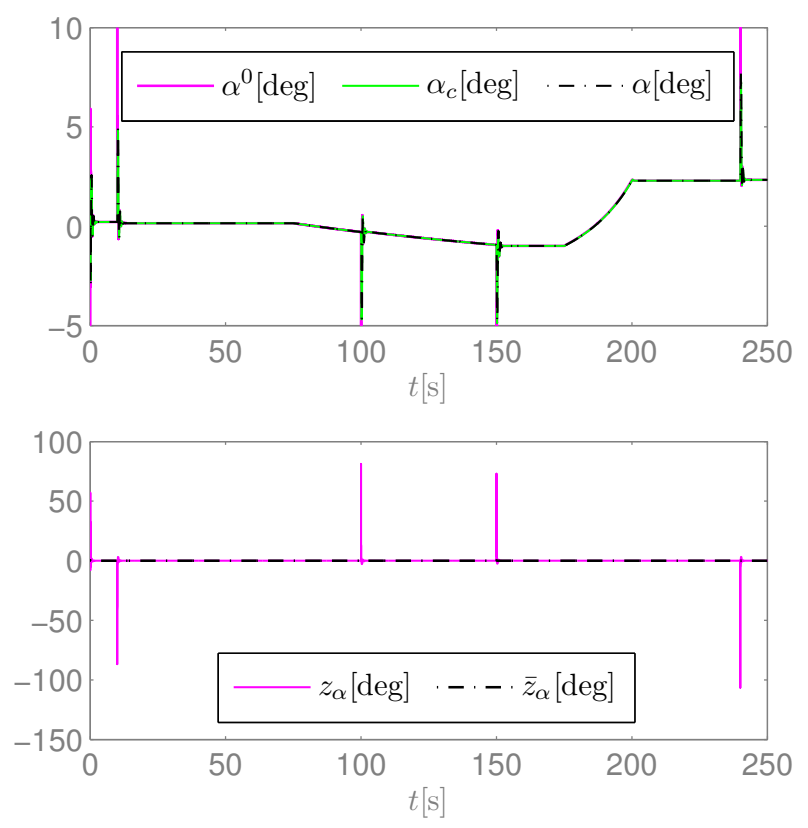

FIGURE 14: $\alpha$ performance and errors.
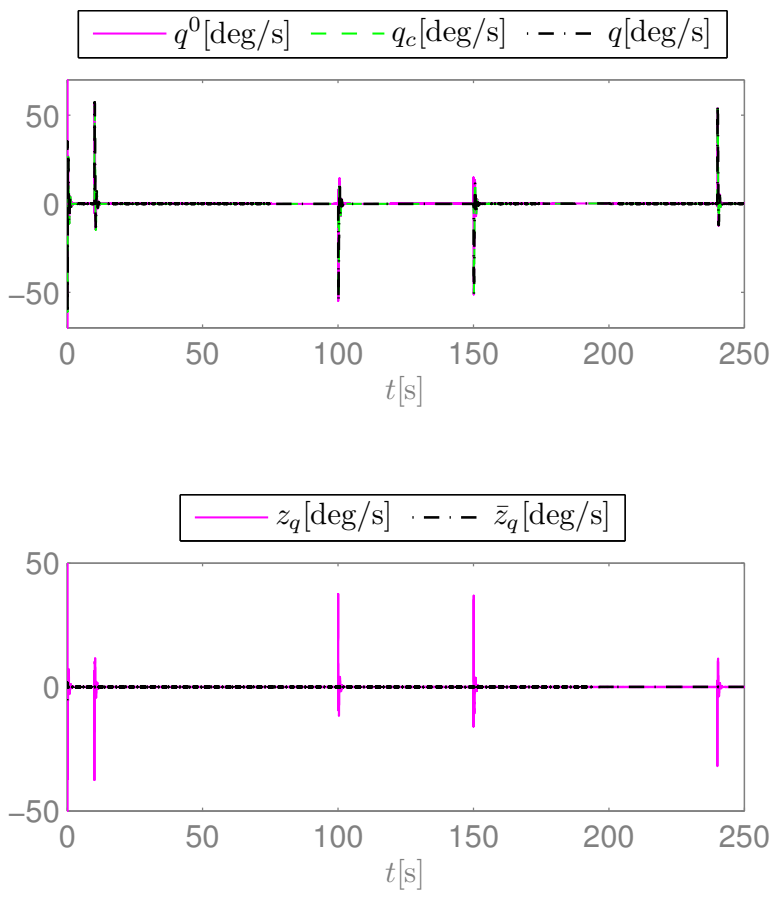

FIGURE 15: $q$ performance and errors. 

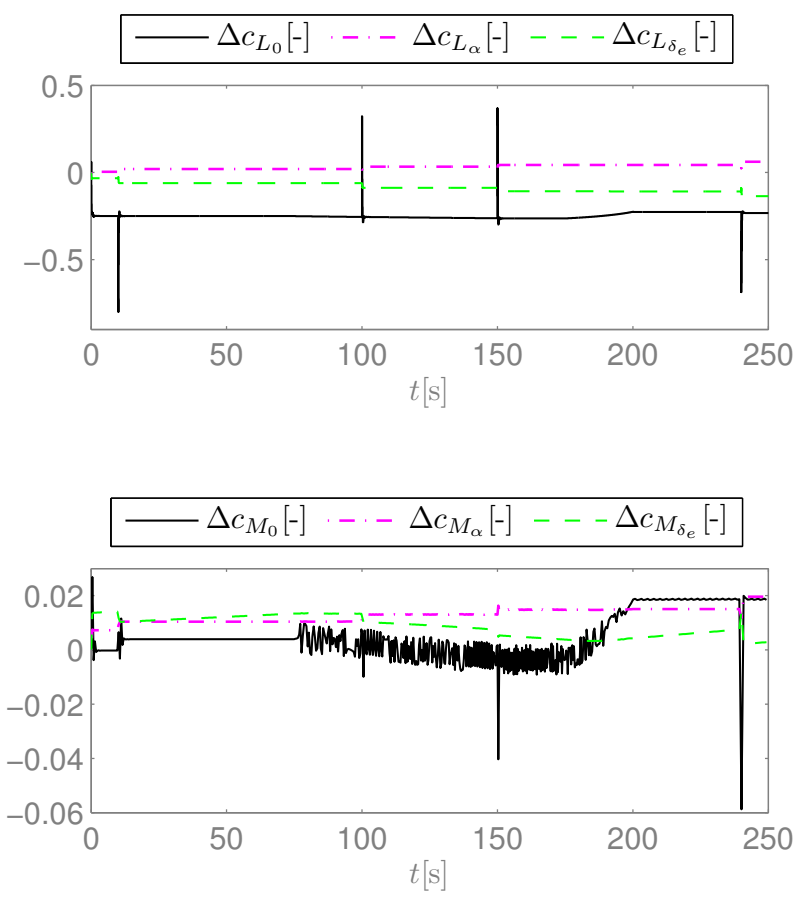

FIGURE 16: Incremental model for $c_{L}$ and $c_{M}$.
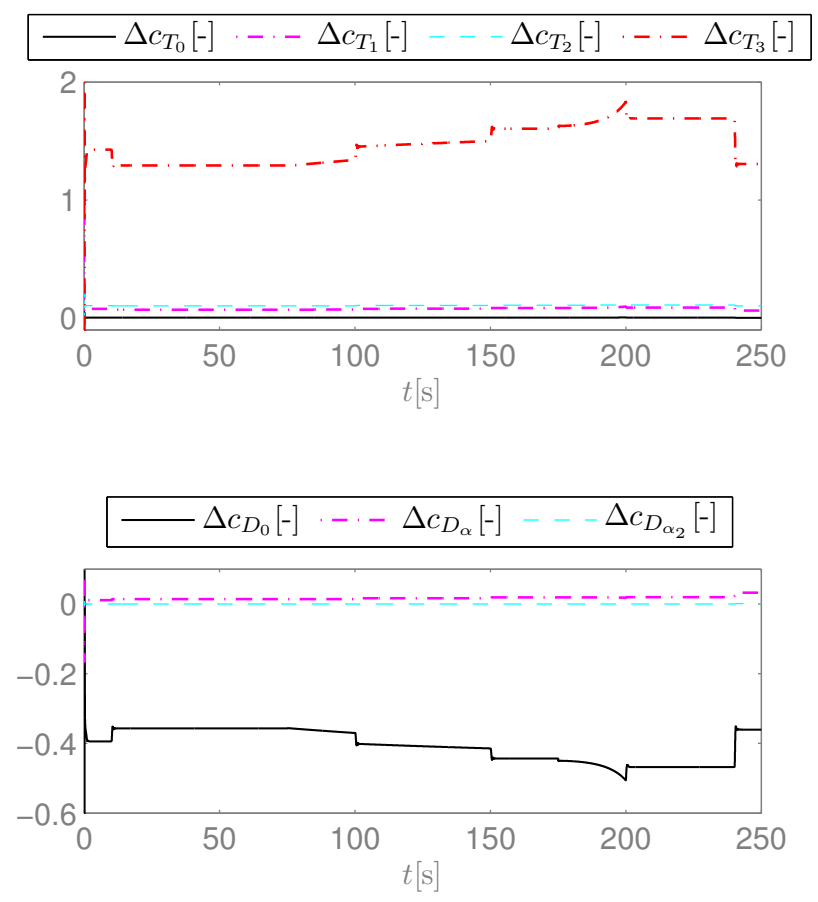

FIGURE 17: Incremental model for $F_{t}$ and $c_{D}$.

An additional study was also carried out to validate the controller when the model of the aircraft that runs in simulation includes the lateral/directional dynamics, concluding that the controller proposed here maintains performance even for extremely adverse coupling effects.

\section{Conclusions}

This paper introduces a clear and systematic process to create a non-linear controller with reliable proofs and high-performance from the modelling process up to the controller derivation.

A comprehensible model of the longitudinal dynamics for the TX-1570 has been derived in the first section of the paper through the definition of a model for the propulsion system based on aerodynamic propeller data, a DC-motor model and a steady-state aerodynamic model. The resulting model is not redundant in control terms and therefore controllability needs to be ensured. An adaptive robust backstepping technique with command filtered virtual control has been applied to derive two controllers in cascade that guarantee the aircraft to track velocity and flight-path angle references within a certain adjustable margin. The projection operator has been implemented in the adaptation procedure of the controller parameters avoiding losing controllability. Finally a simulation exploits the potential of the proposed control technique demonstrating good tracking performance of the controlled aircraft.

\section{References}

[1] XFLR5, Analysis of foils and wings operating at low Reynolds numbers. Guidelines for XFLR5 v6.03, 2011.

[2] APC, Apc propellers data base, 2007-2009. URL: http://www . apcprop.com/v/downloads/PERFILES_WEB/datalist . asp.

[3] Unknown, SOPTs monograph. 1: UAS - Unmanned Aircraft System. About its integration in the non-segregated airspace (in spanish), Spanish Defence department, 2009.

[4] J. Farrell, M. Polycarpou, M. Sharma, Adaptive backstepping with magnitude, rate, and bandwidth constraints: Aircraft longitude control, American Control Conference, 2003. Proceedings of the 20035 (2003) 38983904.

[5] J.A. Farrell, M. Sharma, M. Polycarpou, Backstepping-Based Flight Control with Adaptive Function Approximation, Journal of Guidance, Control, and Dynamics 28 (2005) 1089-1102.

[6] T. Glad, O. Harkegard, Backstepping control of a rigid body, 41st IEEE Conference on Decision and Control 4 (2002) 3944-3945.

[7] I. Kanellakopoulos, P.V. Kokotovic, A.S. Morse, Systematic Design of Adaptive Controllers For Feedback Linearizable Systems, American Control Conference, 1991 (1991) 649-654.

[8] P. Kokotovic, The joy of feedback: nonlinear and adaptive, Control Systems, IEEE 12 (1992) 7-17.

[9] E. Lavretsky, T.E. Gibson, Projection operator in adaptive systems, 2011. arXiv: 1112.4232 .

[10] L. Maodeng, J. Wuxing, M. Malcolm, R.M. Colin, Adaptive backstepping control for optimal descent with embedded autonomy, Aerospace Science and Technology 15 (2011) 589-594.

[11] J. Roskam, Methods for estimating drag polars of subsonic airplanes, University of Kansas Lawrence, Kansas, 1973.

[12] L. Sonneveldt, Q.P. Chu, J.a. Mulder, Nonlinear flight control design using constrained adaptive backstepping, Journal of Guidance, Control, and Dynamics 30 (2007) 322-336.

[13] L. Sonneveldt, E.R. Van Oort, Q.P. Chu, J.a. Mulder, Nonlinear adaptive trajectory control applied to an F-16 model, Journal of Guidance, Control, and Dynamics 32 (2009) 25-39. 